\title{
On the Relation Between Inward Pointing Velocity Conditions
}

\author{
Sofia O. Lopes*, Fernando A. C. C. Fontes ${ }^{\dagger}$ and Maria do Rosário de Pinho ${ }^{\dagger}$ \\ *CMAT e Departamento de Matemática e Aplicações, Universidade do Minho, 4800-058 Guimarães, Portugal. \\ ${ }^{\dagger}$ ISR-Porto, Faculdade de Engenharia, Universidade do Porto, 4200-465 Porto, Portugal
}

\begin{abstract}
Inward pointing velocity conditions are important in the study of whether trajectories remain within sets (the so-called invariance or viability) and also to establish constraint qualifications (CQ) under which necessary conditions of optimality for control problem can be guaranteed to be nondegenerate NCO or normal. In our research on nondegenerate NCO we have studied different types of CQs in the form of inward pointing velocity (IPV) conditions, and, in particular, in which situation one type implies other type of IPV. Such findings are reported in the article.
\end{abstract}

Keywords: Optimal control, maximum principle, state constraints, degeneracy phenomenon.

PACS: $02.30 \mathrm{Xx} ; 02.30 \mathrm{Yy}$

\section{INTRODUCTION}

We start by considering a control problem where the trajectory is constrained to some set $H \subset \mathbb{R}^{n}$.

$$
\begin{aligned}
& \dot{x}(t)=f(t, x, u) \\
& x(0)=x_{0} \\
& x(t) \in H
\end{aligned}
$$

and the trajectory starts at the boundary of the set, i.e.,

$$
x(0) \in \text { bdy } H
$$

Consider also a pair $(x, u)$ of functions $x: \mathbb{R}^{+} \rightarrow \mathbb{R}^{n}, u: \mathbb{R}^{+} \rightarrow \mathbb{R}^{m}$ that is admissible for this problem, that is satisfies (1) and (2). In order to have trajectories that remain within sets, we must have an initial velocity that points to the interior of the set. That is the so-called inward-pointing velocity condition:

$$
\exists \tilde{u} \in \mathbb{R}^{m}: \zeta \cdot f\left(t, x_{0}, \tilde{u}\right) \leq 0 \text { a.e. } t \in[0, \varepsilon] \text { with } \zeta \in N_{H}\left(x_{0}\right) \text {. }
$$

where the normal cone is defined ro be $N_{H}(x)=\operatorname{co}\left\{\lambda \lim \frac{v_{i}}{\left|v_{i}\right|}: \lambda \geq 0, v_{i} \perp C\right.$ at $\left.x_{i}, x_{i} \rightarrow x, v_{i} \rightarrow 0\right\}, \operatorname{co} C$ is the convex hull of a set $C$ and $v \perp C$ if $v=x^{\prime}-x$, where the point $x^{\prime}$ has unique closest point $x$ in $C$.

It was well known that when the set $H$ is smooth and described by a smooth functional inequality then $\zeta$ in (3) is a singleton related with the gradient of the functional. Now consider the optimal control problem consisting of minimizing a cost function $J(x, u)$ such that $(x, u)$ satisfy (1) and (2). The pair $(\bar{x}, \bar{u})$ is called minimizer if it is an admissible for this problem and minimizes the cost functional $J(x, u)$. If the control $u$ is assumed to be piecewise left-continuous functions, the pair $(\bar{x}, \bar{u})$ is called a minimizer in the class of piecewise continuous controls.

The Maximum Principle (MP) for these optimal control problems in which the trajectory starts at the boundary of the set, may not give any useful information when the set of candidate to minimizer is equal to the set of admissible solutions. Therefore, it is important to strengthen the conditions of the MP to avoid this degeneracy.

The importance attached to the development of nondegenerate NCO is illustrated in the history of Mathematical Programming ([1], [2]). The best known conditions are perhaps the Kuhn-Tucker conditions [3]. However theses conditions are only a strengthened form of previous Fritz-John conditions [4].

In optimal control problems, strong forms of the MP can be applied when certain constraint qualifications are satisfied. In the literature, these conditions appear in the form of inward pointing velocity conditions, similar to (3). We can distinguish two types of constraints qualifications (CQ):

Type A : There exists a control that can pull the trajectory away from the state constrained boundary in a neighborhood of the initial time. 
Type B : There exists a control that can pull the trajectory away from the state constrained boundary faster then the optimal control on a neighborhood of the initial time.

Each type has its own merits. The CQ of type B, (see [5], [6]) is not easy to verify in general, because we do not know a priori the optimal control function. However, it is typically applicable to problems with less regularity on the data than the CQ of type A (see [7], [8] and [9]). In particular, the importance of results involving CQ with optimal control is illustrated in [10] when applied to calculus of variations problems.

Here, we are interested in study under which conditions the CQ of type A implies the conditions of type B.

\section{MAIN RESULT}

Through this section, we assume the following hypotheses:

H1 There exists a $L \times B$ measurable function $k(t, u)$ such that $t \mapsto k(t, \bar{u}(t))$ is integrable and

$$
\left\|f(t, x, u)-f\left(t, x^{\prime}, u\right)\right\| \leq k(t, u)\left\|x-x^{\prime}\right\|
$$

for $x, x^{\prime} \in \bar{x}(t)+\delta^{\prime} \mathbb{B}, u \in \mathbb{R}^{m}$ a.e. $t \in[0,1]$.

Furthermore there exist scalars $K_{f}>0$ and $\varepsilon^{\prime}>0$ such that

$$
\left\|f(t, x, u)-f\left(t, x^{\prime}, u\right)\right\| \leq K_{f}\left\|x-x^{\prime}\right\|
$$

for $x, x^{\prime} \in \bar{x}(0)+\delta^{\prime} \mathbb{B}, u \in \mathbb{R}^{m}$ a.e. $t \in\left[0, \varepsilon^{\prime}\right]$.

H2 Assume that $H$ is such that the signed distance function

$$
d(x)=\left\{\begin{array}{l}
-\operatorname{dist}(x, b d y(H)), x \in H ; \\
\operatorname{dist}(x, b d y(H)), \text { otherwise }
\end{array}\right.
$$

is continuously differentiable.

For our problem, in which the trajectory starts at the boundary of the set $H$, the minimizing state trajectory can have three different behaviors:

Case 1 The minimizing state trajectory leaves the boundary immediately and stays away from the boundary for some time, i.e. there exists $r \in(0,1)$ such that $\bar{x}(t) \in$ int $\mathrm{H}, \forall t \in(0, r]$.

Case 2 The minimizing state trajectory remains on the boundary on a neighborhood of the initial time, i.e. there exists $r \in(0,1]$ such that $\bar{x}(t) \in$ bdy $\mathrm{H}, \forall t \in[0, r]$.

Case 3 Neither case 1 nor case 2. For any neighborhood of the initial time, the trajectory enters and leaves the boundary, that is, for any $\varepsilon>0$

$$
A \cap(0, \varepsilon] \neq \emptyset \text { and }[0, \varepsilon] \backslash A \neq \emptyset
$$

where

$$
A=\{t \in[0,1]: d(\bar{x}(t))=0\}
$$

Note that, in Case 3 the trajectory has to enter and leave the boundary an infinite number of times in finite time. If this were not the case, we could pick the instant of time nearer to the origin and consider case 1 or case 2 before the first abandonment or return to the boundary had occurred. 
If the minimizer trajectory is $\bar{x}(t)=-t^{3}-t^{3} \sin \left(\frac{1}{t}\right)$ and $H=\mathbb{R}_{0}^{-}$, we have a problem in which the behavior of the of the minimizer trajectory is the case 3 , as it is illustrated in the figure below.

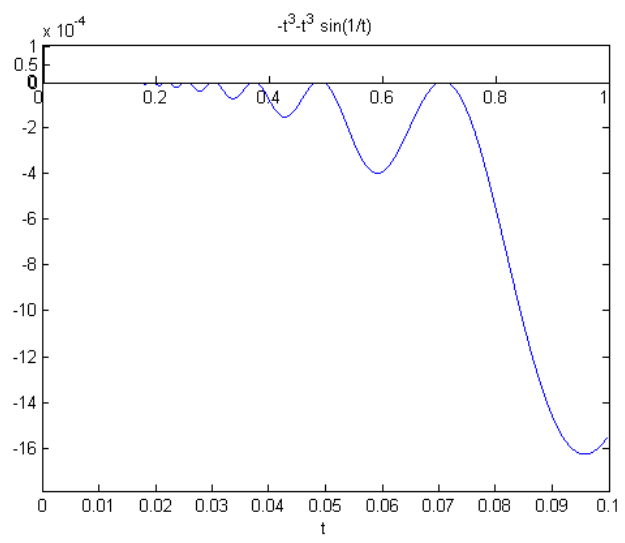

If Case 1 occurs, the MP can be strengthened without assuming any kind of constraint qualifications, see for example [11]. Therefore, in this case, it is useless to compare constraints qualifications.

As mentioned before we can find in the literature two kinds of constraint qualifications:

Type A : For some $\delta>0$

$\exists \tilde{u} \in \mathbb{R}^{m}:$

$$
d_{x}\left(x_{0}\right) \cdot f\left(t, x_{0}, \tilde{u}\right)<-\delta \text { a.e. } t \in[0, \varepsilon) .
$$

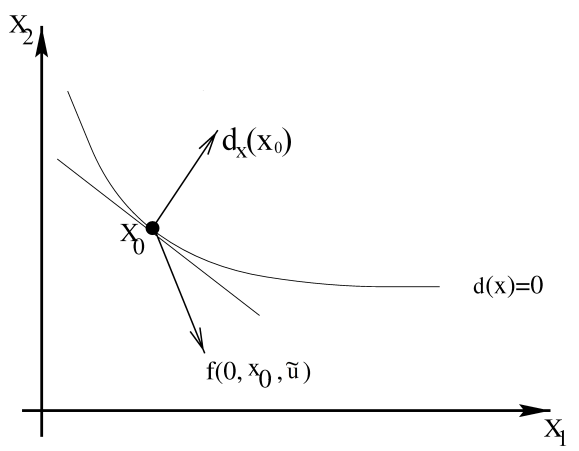

That is, there exists a control that can pull the trajectory away from the state constrained boundary in a neighborhood of the initial time.

Type B : For some $\delta>0$
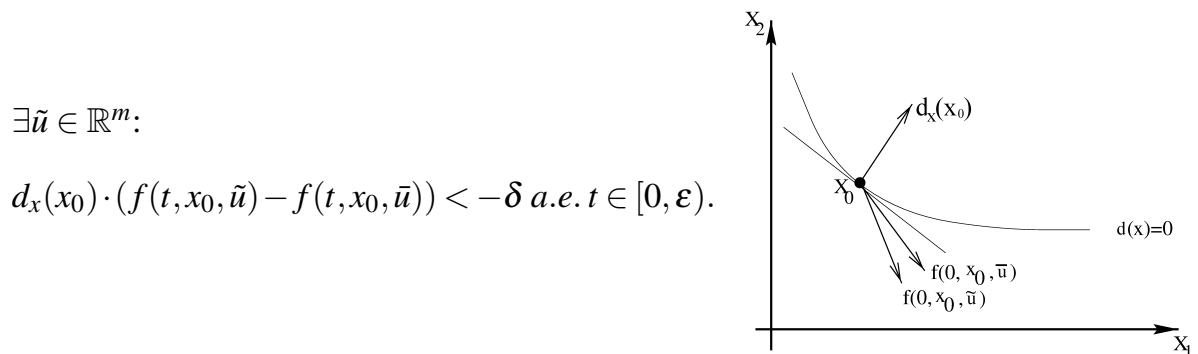

That is, there exists a control that can pull the trajectory away from the state constrained boundary faster then the optimal control on a neighborhood of the initial time.

Proposition 1 Let $(\bar{x}, \bar{u})$ be a minimizer. Assume that hypotheses H1-H2 are satisfied. Additionally, assume that $\exists r \in(0,1]$ :

$$
d(\bar{x}(t))=0, \forall t \in[0, r], \text { (i.e. Case 2). }
$$

Then, CQ of type A implies CQ of type B. 
Idea of the proof: Since $x \rightarrow d(x)$ is continuous differentiable and $x(t)$ is an absolutely continuous function, we have

$$
\frac{d}{d t} d(x(t)) \text { exists for a.e. } t \in[0,1]
$$

For a.e. $t \in[0, r)$, we have

$$
d_{x}(\bar{x}(t)) \cdot f(t, \bar{x}(t), \bar{u}(t))=0 .
$$

Then appealing to $\mathbf{H 1}$ we get the result.

Proposition 2 Let $(\bar{x}, \bar{u})$ be a minimizer in the class of piecewise continuous controls. Assume that hypotheses H1-H2 are satisfied. Let $A=\{t \in[0,1]: d(\bar{x}(t))=0\}$.

If $\forall r \in(0,1)$

$$
A \cap(0, r] \neq \emptyset \text { and }(0, r] \backslash A \neq \emptyset \text {, (i.e. Case 3). }
$$

Then, $C Q$ of type A implies $C Q$ of type $B$.

Idea of the proof: Consider the set $C=(0, r] \backslash A$ and a partition of disjoint opens intervals $\left\{C_{i}\right\}$ such that $C=\cup_{i=1}^{\infty} C_{i}$ with $C_{i}=\left(c_{i}, d_{i}\right), d\left(\bar{x}\left(c_{i}\right)\right)=0, d\left(\bar{x}\left(d_{i}\right)\right)=0$, and $d(\bar{x}(s))<0$ for all $s \in\left(c_{i}, d_{i}\right)$.

We prove by contradiction that there exists $\left.\exists s_{i} \in\right] c_{i}, d_{i}[, \forall i \in \mathbb{N}$ :

$$
d_{x}\left(\bar{x}\left(s_{i}\right)\right) \cdot f\left(s_{i}, \bar{x}\left(s_{i}\right), \bar{u}\left(s_{i}\right)\right) \geq 0 \text {. }
$$

Then using $\mathbf{H 1}$ we get the result.

The complete proof of these propositions can be found in [12].

We conclude that the CQ of type A in the optimal control problems have the disadvantage the optimal control function is required to be be piecewise left-continuous control functions.

On the other hand, our result requires less regularity of the state constraint; $d(\cdot)$ has to be continuously differentiable and not of class $C^{1,1}$, as in the literature.

\section{ACKNOWLEDGMENTS}

The financial support of FCT Project PTDC/EEA-CRO/116014/2009 "Optimal Control in Constrained and Hybrid Nonlinear Systems" and European Commission (FP7-PEOPLE-2010-ITN, Grant Agreement no. 264735-SADCO) is gratefully acknowledged.

\section{REFERENCES}

1. J. Abadie, "On the Kuhn-Tucker Theorem," in Nonlinear Programming, edited by J. Abadie, North Holland, Amsterdam, 1967, pp. 21-56.

2. O. L. Mangasarian, Nonlinear Programming, McGraw-Hill, New York, 1969.

3. H. W. Kuhn, and A. W. Tucker, "Nonlinear Programming," in Proceedings of the Second Berkeley Symposium on Mathematical Statistics and Probability, edited by J. Neyman, University of California Press, Berkeley, 1951, pp. 481-492.

4. F. John, "Extremum Problems as Inequalities as Subsidiary Conditions," in Studies and Essays: Courant Anniversary Volume, edited by K. O. Friedrichs, O. E. Neugebauer, and J. J. Stoker, Interscience Publishers, New York, 1948.

5. M. M. A. Ferreira, and R. B. Vinter, Journal of Mathematical Analysis and Applications 187, $438-467$ (1994).

6. M. M. A. Ferreira, F. A. C. C. Fontes, and R. B. Vinter, Journal of Mathematical Analysis and Applications 233, 116-129 (1999).

7. A. V. Arutyunov, and S. M. Aseev, SIAM Journal of Control and Optimization 35, 930-952 (1997).

8. F. Rampazzo, and R. B. Vinter, SIAM Journal of Control and Optimization 39, 989 (2000).

9. A. Cernea, and H. Frankowska, SIAM Journal of Control and Optimization (2005).

10. S. O. Lopes, and F. A. C. C. Fontes, "Comparison of Nondegenerate Necessary Conditions of Optimality applied to Calculus of Variations Problems," in Proceedings of Controlo 2006, Lisboa, Portugal, 2006.

11. M. M. A. Ferreira, Novo Princípio do Máximo para Problemas de Controlo Óptimo com restricções de Estado, Ph.D. thesis, Falculdade de Engenharia - Universidade do Porto (1994).

12. S. O. Lopes, F. A. C. C. Fontes, and M. do Rosário de Pinho, Discrete and Continuous Dynamical Systems Series A (DCDS-A) 29, 559-575 (2011). 